\title{
Bioavailability of hydrocarbons to bacterial consortia during Triton X-100 mediated biodegradation in aqueous media*
}

\author{
Daria Pęziak1, Aleksandra Piotrowska1, Roman Marecik ${ }^{\bowtie}$, Piotr Lisiecki, Marta Woźniak¹, \\ Alicja Szulc ${ }^{1}$, Łukasz Ławniczak ${ }^{1}$ and Łukasz Chrzanowski ${ }^{1}$ \\ ${ }^{1}$ Institute of Chemical Technology and Engineering, Poznan University of Technology, Poznań, Poland; ${ }^{2}$ Department of Biotechnology and Food \\ Microbiology, Poznan University of Life Sciences, Poznań, Poland
}

\begin{abstract}
The aim of our study was to investigate the effect of Triton X-100 on the biodegradation efficiency of hexadecane and phenanthrene carried out by two bacterial consortia. It was established that the tested consortia were not able to directly uptake compounds closed in micelles. It was observed that in micellar systems the nonionic synthetic surfactant was preferentially degraded (the degradation efficiency of Triton X-100 after 21 days was $70 \%$ of the initial concentration $-500 \mathrm{mg} / \mathrm{l}$ ), followed by a lesser decomposition of hydrocarbon released from the micelles (30\% for hexadecane and $20 \%$ for phenanthrene). However, when hydrocarbons were used as the sole carbon source, $70 \%$ of hexadecane and $30 \%$ of phenanthrene were degraded. The degradation of the surfactant did not contribute to notable shifts in bacterial community dynamics, as determined by Real-Time PCR. The obtained results suggest that if surfactant-supplementation is to be used as an integral part of a bioremediation process, then possible bioavailability decrease due to entrapment of the contaminant into surfactant micelles should also be taken into consideration, as this phenomenon may have a negative impact on the biodegradation efficiency. Surfactant-induced mobilization of otherwise recalcitrant hydrocarbons may contribute to the spreading of contaminants in the environment and prevent their biodegradation.
\end{abstract}

Key words: biodegradation, bioavailability, hexadecane, phenanthrene, Triton X-100

Received: 15 October, 2013; revised: 04 December, 2013; accepted: 04 December, 2013; available on-line: 29 December, 2013

\section{INTRODUCTION}

Several strategies have been proposed in order to overcome the bioavailability limitations in bioremediation. Among them, the addition of surfactants has been recognized as a potentially promising solution. Currently, surfactant supplementation remains one of the most commonly used methods to deal with the difficulties of hydrocarbon degradation (Makkar \& Rockne, 2003). Surfactants are capable of enhancing the apparent solubility by forming aggregates in water called micelles. The process of creating micelles occurs above the critical micelle concentration value (CMC), and depends on type and concentration of the surfactant. Over the last few years, attention has been focused on the use of biosurfactants to supplement the bioremediation process (Lawniczak et al., 2013). However, the synthetic anionic and nonionic surfactants are still commonly employed in order to fa- cilitate the utilization of organic contaminants, mostly due to economical issues. Particularly, the ethoxylated nonionic surfactants have been commonly applied in bioremediation (Guha \& Jaffe, 1996a). The major reason is the price and a favorable behavior with regard to temperature, which allows to carry out the biodegradation process under environmental conditions (e.g. at a temperature of $10^{\circ} \mathrm{C}$ ) (Zeng et al., 2007). The representative of mentioned group is Triton X-100, a commercial name for a group of ethoxylated p-tert-octyl phenol homologues.

Although the supplementation of surfactants in degradation processes has become a popular practice, there are still many questions and unsolved problems associated with actual influence of such substances on biodegradation. The results of several biodegradation studies regarding the effect of surfactant-supplementation are ambiguous, as different scientific reports claim that positive, neutral and even negative influence of surfactant on biodegradation may be observed. For example, Dai et al. (2010) observed that organic compounds solubilized in micellar phase are unavailable for bacteria. Overall, although the mechanisms of interactions between bacterial cells and surfactants have been investigated, the exact influence of surface-active agents on the biodegradation process is hard to evaluate and therefore challenging to predict (Wang, 2011). Thus, the topic of surfactant supplementation raises many doubts and it is worthy of further research.

The aim of this study was to investigate the bioavailability of hexadecane and phenanthrene in Triton X-100 micelles and the subsequent effect on their biodegradation efficiency by diesel oil-degrading bacterial consortia. This was achieved by comparing the results of hydrocarbon loss in samples with and without Triton X-100 supplementation. Additionally, the experiments also focused on assessing the possible shifts in bacterial community dynamics.

\section{MATERIALS AND METHODS}

Bacterial consortia. Two bacterial consortia (K52 and G23) previously isolated from crude oil-contaminated soil (Czarna and Gorlice, Małopolska, Poland) have been used in this study. The members of the K52

e-mail: romarc@up.poznan.pl

* Presented at the 5th Central European Congress of Life Sciences „EUROBIOTECH 2013”, Kraków, Poland. 
consortium were identified in the framework of another study (Owsianiak et al., 2009a) as the following bacterial taxa: Achromobacter sp., Alcaligenes sp., Citrobacter sp., Comamonadaceae, Pseudomonas sp., Sphingobacterium sp., V ariovorax sp.

The members of the G23 consortium were determined in the framework of this study based on the method described in Wyrwas et al. (2011) and belonged to the following taxa: Achromobacter xylosoxidans, Alteromonas sp., Burkholderia cepacia, Enterobacter cloacae, Ochrobactrum anthropi, Pseudomonas aeruginosa, Pseudomonas fluorescens, Sphingomonas sp., Stenotrophomonas maltophilia, Xanthomonas oryzae. The results of previous studies showed that both K52 and G23 displayed a notable initial diesel oil biodegradation potential, which was decreased upon the addition of Triton X-100. The reason of using two bacterial consortia (K52 and G23) during the experiment was to assess whether the presence of specific microbial taxa may contribute to different interactions with Triton X-100.

Chemicals. Carbon sources. Two types of hydrocarbons, phenanthrene and hexadecane, were used in the experiments. Both phenanthrene and hexadecane were purchased from POCh, Gliwice.

Surfactants. In our studies the commercial Triton $\mathrm{X}-100$ (Sigma, Aldrich) has been used. It is a mixture of alkylphenol ethoxylates (APE) with an average degree of ethoxylation equal to 9.5 .

Biodegradation experiments. The biodegradation testes were carried out in 48 sterile flasks $(200 \mathrm{ml})$. Half of the samples contained $50 \mathrm{mg}$ of the hexadecane, the other half contained $5 \mathrm{mg}$ of phenanthrene dissolved in acetone. Upon introduction of phenanthrene the solvent was evaporated. The hydrocarbon doses used during the studies were based on the Triton X-100 MSR value and the concentrations of hydrocarbons commonly used in biodegradation processes (Paria, 2008; Yang et al., 2009). Next, $50 \mathrm{ml}$ of mineral medium (composition described in Owsianiak et al., 2009b) supplemented with Triton X-100 were introduced into 18 flasks (9 for each carbon source). The amount of Triton X-100 (500 mg/l) introduced into the samples was calculated so that it would allow for a complete solubilization of both hydrocarbons. Afterwards, $50 \mathrm{ml}$ of the mineral medium without the surfactant were added into the remaining 18 flasks in order to obtain a parallel set for each respective hydrocarbon. During the last step each flask was inoculated with the corresponding bacterial consortium (half of flasks with K52 and the other half with G23, respectively). The biomass amount for each inoculum was set up to an $\mathrm{OD}_{600}$ of $0.1 \pm 0.01$. An additional set of blanks was used to evaluate the abiotic losses for each experimental set-up. After that, the flasks were shaken at $180 \mathrm{rpm}$ and $10^{\circ} \mathrm{C}$. The samples were taken after $7^{\text {th }}$, $14^{\text {th }}$ and $21^{\text {st }}$ days for subsequent analysis of hydrocarbon loss and changes in the Triton X-100 concentration.

Analytical procedures. GC-FID hydrocarbons determination. The HRGC Mega Series 53000 chromatograph with the cold on column injector and flame ionization detector were used. Analyses were performed on capillary columns with nonpolar ( $5 \%$ phenyl)-methylpolysiloxane (HP-5) stationary phases - $25 \mathrm{~m} \times 0.32$ $\mathrm{mm}$. The helium pressure was $85 \mathrm{kPa}$. The column temperature was held at $60^{\circ} \mathrm{C}$ for 2 minutes and ramped at $25^{\circ} \mathrm{C} / \mathrm{min}$ to $160^{\circ} \mathrm{C}$, next the temperature was raised with a rate $15^{\circ} \mathrm{C} / \mathrm{min}$ to the final temperature $260^{\circ} \mathrm{C}$.

HPLC/ESI-MS analysis of Triton X-100 residues. The ethoxylates were separated from water samples by sequential extraction with ethyl acetate (APE) and chloroform (PEG) as previously described by Szymanski and
Lukaszewski (1996). The extracts aliquots were used for analyses. The HPLC/ESI-MS analyses were performed using a UltiMate 3000 RSLC (Dionex Sunnyvale, CA, USA), coupled with 4000 QTRAP LC/MS/MS Hybrid Triple Quadrupole/Linear Ion trap mass spectrometry (AB SCIEX Foster City, CA, USA), Hypersil GOLD column $(1.9 \mu \mathrm{m} \times 100 \mathrm{~mm} \times 2.1 \mathrm{~mm}$, Thermo Fisher Scientific Inc.) according to the procedure described in Wyrwas et al. (2011).

PCR-based analysis of bacterial community dynamics. Analysis of changes in the abundance of specific microbial taxa was carried out with the use of quantitative real-time PCR procedure described in Cyplik et al. (2011).

\section{RESULTS AND DISCUSSION}

\section{Biodegradation of hydrocarbons and possible mechanisms of hydrocarbon uptake in the presence of Triton X-100}

The results obtained during biodegradation of hexadecane and phenanthrene were showed at Figs 1 and 2, respectively. It could be observed that the biodegradation of hexadecane (straight-chain hydrocarbon), was faster than the biodegradation of phenanthrene (aromatic hydrocarbon). The biodegradation efficiencies were similar for both consortia in samples with and without Triton $\mathrm{X}-100$. The biodegradation process was carried out at the temperature of $10^{\circ} \mathrm{C}$ (conditions similar to those in a cold temperature climate environment for middle Europe). It is worth noticing that the hydrocarbon biodegradation rate was lower in surfactant-supplemented samples for both consortia. The hexadecane loss values during the first 7 days were below 0 due to poor recovery efficiency (Fig. 1). Hypothetically, the actual value during this period was close to 0 , hence the actual biodegradation occurred between the 7th and 14th day. At the 21st day of the experiment (the last day), it could be noticed that the hexadecane removal efficiency reached the value of approx. $70 \%$ for samples without the presence of surfactant, in comparison to approx. $20 \%$ achieved for samples containing Triton X-100. This tendency was similar for samples where phenanthrene was the intended source of carbon. The biodegradation rate was higher

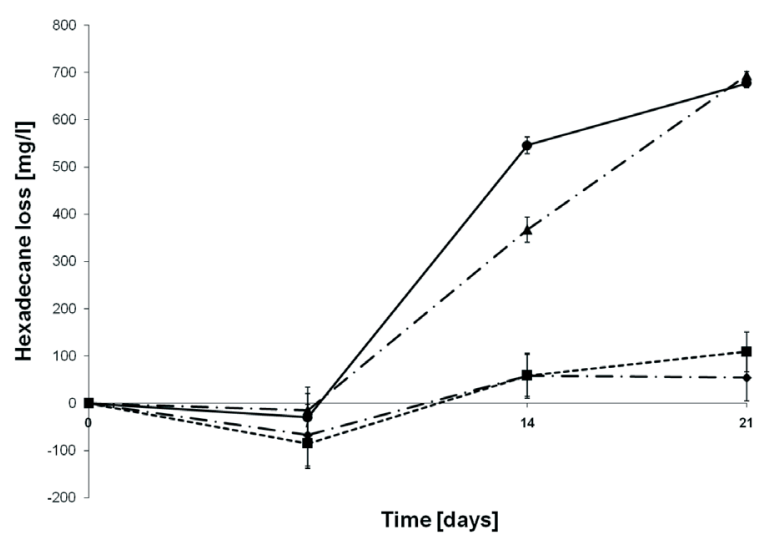

Figure 1. The loss of hexadecane (initial dose at $1000 \mathrm{mg} / \mathrm{L}$ ) from samples with and without Triton X-100.

Monitored during 21 days of biodegradation experiment with the use of consortia K52 and G23 ( $\boldsymbol{\Delta}$, system without Triton X-100 with consortium K52; 0 , system without Triton X-100 with consortium G23; $\bullet$, system with Triton X-100 with consortium K52; system with Triton X-100 with consortium G23). 


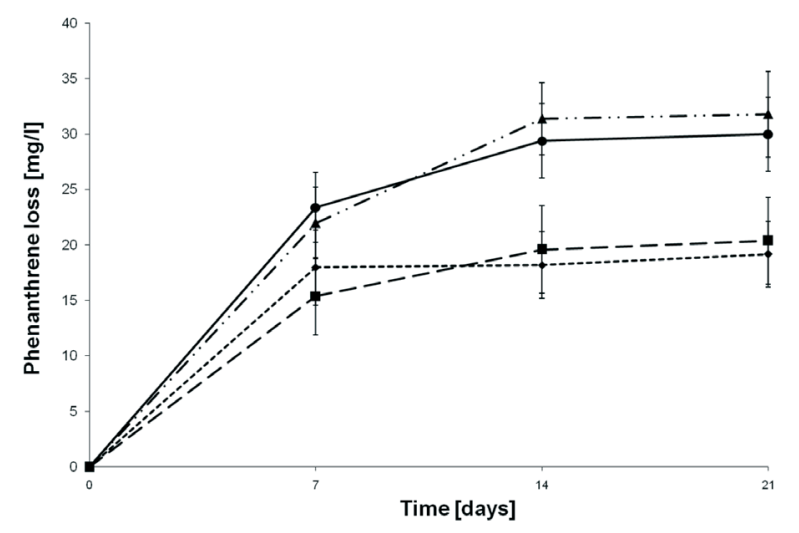

Figure 2. The loss of phenanthrene (initial dose at $100 \mathrm{mg} / \mathrm{L}$ ) from samples with and without Triton X-100.

<onitored during 21 days of biodegradation experiment with the use of consortia K52 and G23 ( $\boldsymbol{\Delta}$, system without Triton X-100 with consortium K52;, , system without Triton X-100 with consortium G23; $\bullet$, system with Triton X-100 with consortium K52; system with Triton X-100 with consortium G23).

for samples without addition of surfactant, although the difference was not as notable as for the hexadecane experiment. The phenanthrene loss reached approximately $30 \%$ of the initial concentration in the absence of Triton $\mathrm{X}-100$, while in the presence of Triton X-100 the phenanthrene content decreased by $20 \%$.

It was established that the bioavailability of substrates to microorganisms was the main limiting factor for the biodegradation process. Upon the addition of surfactant into the samples, the surface-active amphiphilic molecules deposited at interface between the organic contaminants and the aqueous phase. This prevented the contact between bacterial cells and the organic phase. Hence, the hydrocarbon uptake would be inhibited. In order to reach the hydrocarbons, the surfactant would have to be firstly degraded by bacteria, which resulted in the elongation of the whole biodegradation process. After the microorganisms got access to the hydrocarbons, the biodegradation could be continued.

Alternatively, as suggested by other researchers, the decrease of the bacterial adherence to hydrocarbons after addition of surfactant may have also inhibited the biodegradation process (Efroymson \& Aleksander, 1991; Stelmack et al., 1999). In our studies this aspect was not investigated and this explanation cannot be excluded.

\section{Community dynamics and toxicity of Triton X-100}

In our biodegradation experiment, two specific experimental set-ups with and without the addiction of Triton X-100 (concentration corresponded to approx. 3.5 times the CMC value) were created. The presence of a high surfactant concentration might have caused shifts in the bacterial community dynamics. Therefore in the next experimental step, the K52 consortium used in this study was subjected to Real-Time PCR in order to evaluate whether the surfactant had any influence on the structure of the studied consortia. The results were presented on Fig. 3. During growth on hexadecane (Fig. 3A) the changes in abundance of specific consortium members were within the same order of magnitude for samples with and without surfactant addition. This suggested that the presence of Trion X-100 had no notable influence on the bacterial consortia, and that the growth of each consortium member was not disturbed. In the case of growth on phenanthrene (Fig. 3B) the only difference was a slightly more intensive growth of Comamonadaceae sp. and Sphingobacterium sp. in the samples without Triton $\mathrm{X}-100$.

The results from Real-Time PCR tests obtained during 21 days of studies showed that the abundance of all members of consortium K52 increased compared to the initial state. This proves that Triton X-100 did not have a toxic effect on bacterial species like: Achromobacter sp., Alcaligenes sp., Citrobacter sp., Comamonadaceae, Sphingobacterium sp., Psendomonas sp., Variovorax sp.. The same conclusion was reported by Volkering et al., (1995). The authors claimed that the applied concentration of Triton X-100 did not have a toxic effect on the studied microorganisms. It was suggested that even at $10 \mathrm{~g} / \mathrm{L}$ Triton $\mathrm{X}-100$ was not toxic towards most bacterial species. In our case, the toxic effect was not observed, suggesting that this phenomenon may be specific towards certain bacterial species.

However, it is also worth noticing that the obtained results suggest that none of the studied bacterial taxa demonstrated preferential growth on hexadecane (the relative increase in abundance for each specific member was comparable, no single taxa dominated). As such, no bacterial species specialized in the degradation of hexadecane or Triton X-100 could not be distinguished. Increased abundance of Sphingobacterium sp. during biodegradation of phenanthrene may suggest that this bacterial taxa prefers this specific carbon source. This is in accordance with other studies, as reports regarding the high biodegradation potential of Sphingobacterium sp. towards PAH can be found (Alias et al., 2012).
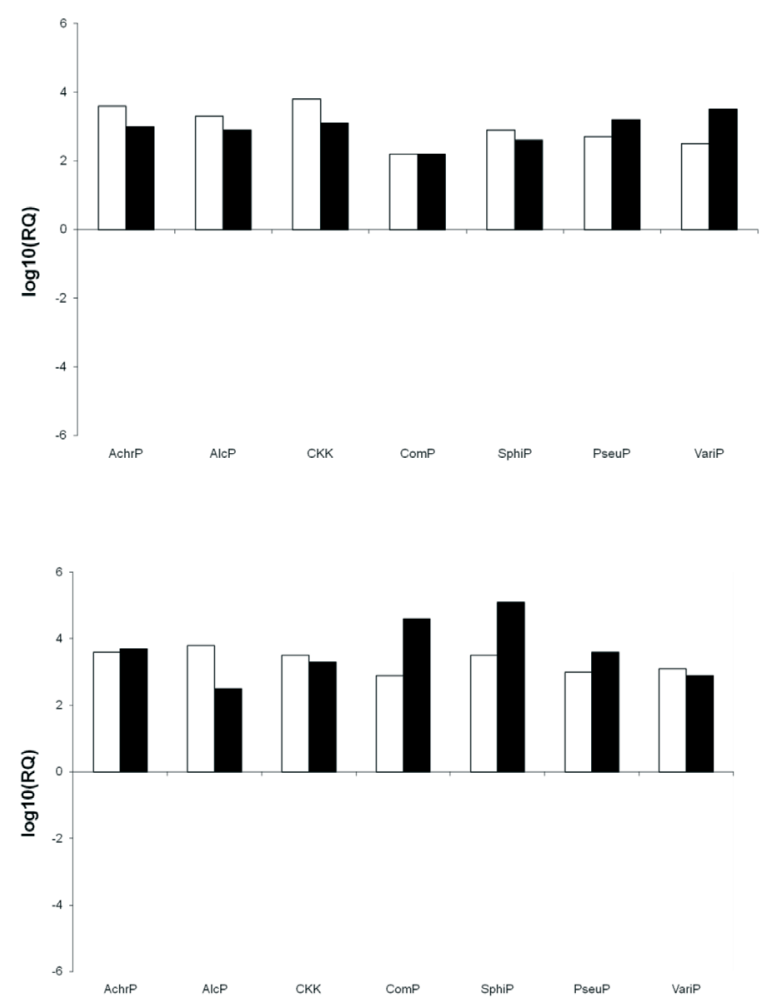

Figure 3. Changes in bacterial community as monitored by relative quantitative real-time PCR during biodegradation of hexadecane $(A)$ and phenanthrene $(B)$ in a system with Triton X-100 (white) and without it (black).

The members of consortium K52 were grown for 21 days and results for each bacterial group quantitation were normalized to the total number of bacteria: Achromobacter $s p$. (AchrP), Alcaligenes $s p$. (AlcP), Citrobacter sp. (CKK), Comamonadaceae (ComP), Sphingobacterium $s p$ (SphiP), Pseudomonas $s p$. (PseuP), Variovorax sp. (VariP). 


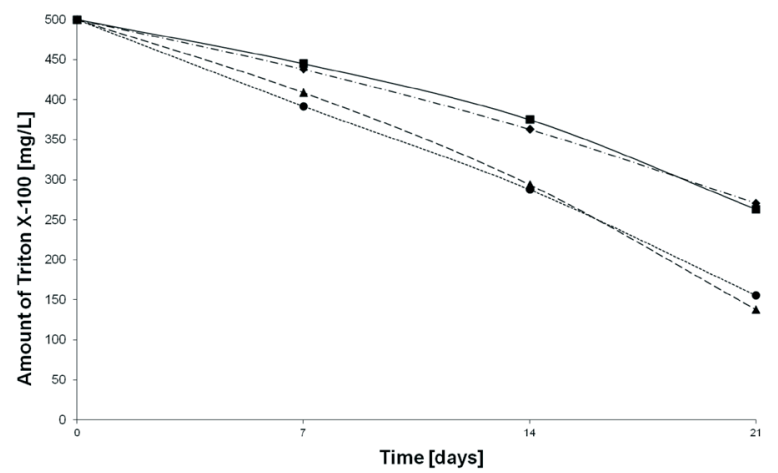

Figure 4. Changes in Triton X-100 concentration (initial dose at $500 \mathrm{mg} / \mathrm{L}$ ) monitored during 21 days of biodegradation experiment with the use of consortia K52 and G23

( $\boldsymbol{\Lambda}$, degradation of Triton X-100 solubilized hexadecane conducted by consortium G23; , degradation of Triton X-100 solubilized phenanthrene conducted by consortium G23; $\bullet$, degradation of Triton X-100 solubilized hexadecane conducted by consortium K52; , degradation of Triton X-100 solubilized phenanthrene conducted by consortium K52).

Overall, the bacterial community dynamics experiment proved that the structure of the K52 consortium was stable during degradation of hydrocarbons with and without the addition of Triton X-100.

\section{The utilization of Triton X-100}

Another possible way to explain the retardation of hydrocarbon biodegradation process due to the addition of surfactant could be the preferential utilization of Triton $\mathrm{X}-100$ as a carbon source. To verify this hypothesis a measurement of surfactant concentration changes was carried out and compared. Shifts in the concentration of Triton X-100 during the biodegradation process were presented in Fig. 4. The obtained results suggest that the degradation of surfactant occurred, since the concentration of surfactant in samples with phenanthrene and hexadecane decreased. Additionally, the members of the G23 consortium were better surfactant degraders compared to members of the K52 consortium. At the 21 -st day of experiment the amount of biodegraded Triton $\mathrm{X}-100$ exceeded $70 \%$ of the initial concentration for consortium G23 and about 40\% for the samples with consortium K52. It could be observed that the type of solubilized compound had no influence on the biodegradation of Triton X-100. The results regarding the preferential utilization of surfactant molecules are in accordance with other scientific reports (Graves \& Leavitte, 1991, Zeng et al., 2007; and Wyrwas et al., 2011).

On the contrary, the results obtained by Guha and Jaffe showed that an enhancement of biodegradation processes upon supplementation with nonionic surfactant was achieved. The authors used the experimental data to create a mathematic model and stated that phenanthrene was directly bioavailable for microorganisms from micelles, however not for all investigated nonionic surfactants (Guha \& Jaffe, 1996a; Guha \& Jaffe, 1996b). Furthermore, it was suggested that micelles interacted with bacterial cells. The authors claimed that the bioavailability factor likely depended on the type of surfactant and the nature of a given bacterial culture, which is highly plausible when considering that the experiments were carried out with the use of monocultures. The fact, that the authors also suggested that the bioavailability of contaminants would decrease if the dose of surfactant increased in the same time is of importance. It was established that the addition of a high surfactant dose could decrease the concentration of organic compounds dissolved in the aqueous phase and result in limited mass transfer from micelles to cells. This statement corresponds well with the results presented in our studies, as both carbon sources became unavailable for bacterial cells in the presence of Triton X-100.

Overall, the results obtained in the framework of this studies lead to several conclusions. Firstly, phenanthrene and hexadecane during the biodegradation process mediated by Triton X-100 were not degraded efficiently. Similar conclusion was also reported by Dai et al. (2010). The presence of micelles increased the solubility of hydrophobic compounds, thus hypothetically enhancing their bioavailability, however it also created a barrier between the bacteria and the solubilized hydrocarbons. This corresponds well with the observations regarding decrease of bioavailability for hydrophobic compounds in micelles reported by Chrzanowski et al. (2009; 2011) during studies on chlorinated phenols solubilized in ramnolipids micelles. On the other hand, it is also plausible that the degradation of hydrocarbons supported by Triton X-100 is possible (i.e. flushing of contaminated soil followed by subsequent biodegradation of the waste stream), if controlled properly. The main reason for the observed retardation of biodegradation efficiency could be preferential utilization of Triton X-100 by bacteria as a carbon source.

\section{Acnowledgements}

This study was supported by NCN grant: OPUS 2 2011/03/B/NZ9/00274.

\section{REFERENCCES}

Alias S, Hussain N-H, Omar M, Abdul-Talib S (2012) Biodegradation of high-molecular-weight polycyclic aromatic hydrocarbon in contaminated sand by Sphingobacterium spiritovorum and Corynebaterium urealyticum. Int J Eng Phys Sci 6: 369-376.

Broze G (1999) Handbook of Detergents Part A: Properties. Surfactant Science Series 82.

Chrzanowski Ł, Owsianiak M, Szulc A, Marecik R, Piotrowska-Cyplik A, Olejnik-Schmidt AK, Staniewski J, Lisiecki P, Ciesielczyk F, Jesionowski T, Heipieper HJ (2011) Interactions between rhamnolipid biosurfactants and toxic chlorinated phenols enhance biodegradation of a model hydrocarbon-rich effluent. Int Biodeter Biodegr 65: 605-611.

Chrzanowski Ł, Wick LY, Meulenkamp R, Kaestner M and Heipieper HJ (2009) Rhamnolipid biosurfactants decrease the toxicity of chlorinated phenols to Pseudomonas putida DOT-T1E. Lett Appl Microbiol 48: 756-762.

Cyplik P, Schmidt M, Szulc A, Marecki R, Lisiecki P, Heipieper HJ, Owsianiak M, Vainshtein M, Chrzanowski $\ell$ (2011) Relative quantitative PCR to assess bacterial community dynamics during biodegradation of diesel and biodiesel fuels under various aeration conditions. Biores Technol 102: 4347-4352.

Dai Z, Wang Z, Xu J-H Qi H (2010) Assessing bioavailability of the solubilization of organic compound in nonionic surfactant micelles by dose-response analysis. Appl Microbiol Biotechnol 88: 327-339.

Das N, Chandran P (2011) Microbial degradation of petroleum hydrocarbon contaminants. Biotechnol Res Int 2011: 1-13.

Efroymson R, Aleksander M (1991) Biodegradation be an Arthrobacter species of hydrocarbons partitioned into an organic solvent. Appl Environ Microbiol 57: 1441-1447.

Graves D, Leavitt M (1991) Petroleum biodegradation in soil: The effect of direct application of surfactants. Remediation 1: 147-166.

Guha S, Jaffe PR (1996) Biodegradation kinetics of phenantrene partitioned into the micellar phase of nonionic surfactants. Environ Sci Technol 30: 605-611.

Guha S, Jaffe PR, (1996) Bioavailable of hydrophobic compounds partitioned into the micellar phase of nonionic surfactants. Environ Sci Technol 30: 1382-1391.

Lawniczak L, Marecik R, Chrzanowski L, (2013) Contributions of biosurfactants to natural or induced bioremediation. Appl Microbiol Biotechnol 97: 2327-2339. 
Makkar RS, Rockne KJ (2003) Comparison of synthetic surfactants and biosurfactants in enhancing biodegradation of polycyclic aromatic hydrocarbons. Environ Toxicol Chem 22: 2280-2292.

Owsianiak M, Szulc A, Chrzanowski L, Cyplik P, Bogacki M, OlejnikSchmidt AK, Heipieper HJ (2009a) Biodegradation and surfactantmediated biodegradation of diesel fuel by 218 microbacterial consortia and not correlated to cell surface hydrophobicity. Appl Microbiol Biotechnol 84: 545-553.

Owsianiak M, Chrzanowski L, Szulc A, Staniewski J, Olszanowski A, Olejnik-Schmidt AK, Heipieper HJ (2009b) Biodegradation of diesel/biodiesel blends by a consortium of hydrocarbon degraders: Effect of the type of blend and the addition of biosurfactants. Bioresource Technol 100: 1497-1500.

Paria S (2008) Surfactant — enhanced remediation of organic contaminanted soil and water. Adv Collloid Interface Sci 138: 24-58.

Stelmack PL, Gray MR, Pickard MA (1999) Bacterial adhesion to soil contaminants in the presence of surfactants. Appl Environ Microbiol 65: 163-168.

Szymanski A, Lukaszewski Z (1996) Determination of poly(ethylene glycols) in environmental samples by the indirect tensammetric method. Analyst 121: 1897-1901.
Wang Z (2011) Bioavailability of organic compounds solubilized in nonionic surfactant micelles. Appl Microbiol Biotechnol 89: 523-534.

Willumsen A, Karlson U, Pritchard PH (1998) Response of fluoranthene-degrading bacteria to surfactants. Appl Microbiol Biotechnol 50: 475-483.

Wyrwas B, Chrzanowski Ł, Lawniczak L, Szulc A, Cyplik P, Białas W, Szymański A, Hołderska-Odachowska A (2011) Utilization of Triton X-100 and polyethylene glycols during surfactant-mediated biodegradation of diesel fuel. J Hazard Mater 197: 97-103.

Volkering F, Breure AM, Andel JG, Rulkens WH (1995) Influence of nonionic surfactants on bioavailability and biodegradation of polycyclic aromatic hydrocarbons. Appl Environ Microbiol 61: 1699-705.

Yang J, Chen JJ, Lu Y (2009) Removal mechanism of lighf non-aqueus phase liquid from soil and groundwater by surfactants. China Environ Sci 30: 2153-2159.

Zeng G, Fu H, Zhong H (2007) Co-degradation with glucose of four surfactants, CTAB, Trition X-100, SDS and Rhamnolipid, in liquid culture media and compost matrix. Biodegradation 18: 303-310. 